FACTA UNIVERSITATIS

Series: Mechanical Engineering Vol. 15, No 1, 2017, pp. 31 - 44

DOI: $10.22190 /$ FUME170225002R

Original scientific paper

\title{
A 3-NODE PIEZOELECTRIC SHELL ELEMENT FOR LINEAR AND GEOMETRICALLY NONLINEAR DYNAMIC ANALYSIS OF SMART STRUCTURES
}

\author{
UDC $624.01+531 ; 519.6$
}

\author{
Gil Rama \\ Berlin Institute of Technology (TU Berlin), \\ Department of Structural and Computational Mechanics, Germany
}

\begin{abstract}
Composite laminates consisting of passive and multi-functional materials represent a powerful material system. Passive layers could be made of isotropic materials or fiber-reinforced composites, while piezoelectric ceramics are considered here as a multifunctional material. The paper is focused on linear and geometrically nonlinear dynamic analysis of smart structures made of such a material system. For this purpose, a linear 3node shell element is used. It employs the Mindlin-Reissner kinematics and the discrete shear gap (DSG) technique to alleviate the transverse shear locking effects. The electric potential is assumed to vary linearly through the thickness for each piezoelectric layer. A co-rotational formulation is used to handle the geometrically nonlinear effects. A number of examples involving actuator and sensor application of piezoelectric layers are considered. For the validation purposes, the results available in the literature and those computed in Abaqus are used as a reference.
\end{abstract}

Key Words: Shell Element, Piezoelectricity, Active Laminates, Co-rotational FEM, Actuator, Sensor, Geometrically Nonlinear Dynamic

\section{INTRODUCTION}

Laminated thin-walled structures made of isotropic or orthotropic materials are widely used in engineering practice. This is a consequence of the optimization strategy to reduce the structural dead-load whereby the structural carrying capacity is kept at a very high level. Besides numerous advantages offered by thin-walled structures, they also tend to suffer from structural stability issues and are rather sensitive to vibrations. The use of multi-functional materials offers a great potential to cope up with those challenges.

Received: February 25, 2017 / Accepted March 21, 2017

Corresponding author: Gil Rama

TU Berlin, Fachgebiet für Strukturmechanik und -berechnung, Str. des 17. Juni 135, 10623 Berlin, Germany

E-mail: gil.rama@tu-berlin.de 
Piezoelectric materials are characterized by a sufficiently strong coupling between the mechanical and the electric fields, so that they can be employed for an adequately designed actuator as well as sensor devices. In the actuator case, the inverse piezoelectric effect is used to affect the mechanical field through a purposeful change of the electric field. Oppositely, the direct piezoelectric effect is used for sensors to gain information on the induced deformation, i.e. strain-field in the material.

The Finite Element Method (FEM) has established itself as the method of choice in the field of structural analysis including coupled-field problems, such as the piezoelectric effect. Over the last couple of decades, numerous elements have been developed for static and dynamic analyses of piezoelectric thin-walled structures. A survey of piezoelectric solids, beams, plates and shells developed in the 90's is given by Benjeddou [1]. Solid elements, such as those proposed by Lee et al. [2] and Willberg and Gabbert [3], provide high fidelity FE modeling but at the price of a high numerical effort when applied to thin-walled composites. Therefore, shell type finite elements are usually addressed as numerically more efficient for this type of structures when the global structural behavior is aimed at.

Most of the composite shell FE formulations are based on the equivalent single-layer approach and mainly rely on the Kirchhoff-Love or Mindlin-Reissner kinematics. The Kirchhoff-Love kinematics leads to zero transverse shear strains/stresses and is therefore applicable to rather thin shells. The Mindlin-Reissner kinematics takes the transverse shear strains into account, so that the resulting theory is referred to as the first-order shear deformation theory (FSDT). The Mindlin-Reissner plate and shell elements are notorious for shear locking when rather thin structures are modeled. Various techniques have been developed to eliminate the effect, such as the Assumed Natural Strain (ANS) [4], Enhanced Assumed Strain (EAS) [5], reduced integration schemes and the Discrete Shear Gap (DSG) method [6]. All of them were also used in the development of piezoelectric shell elements. Marinković et al. [7] developed a full biquadratic degenerated shell element with a choice between the full and uniformly reduced integration scheme. The element was used to check the convergence of FE results for the coupled electro-mechanical field [8] and it was also implemented in Abaqus [9] for the users' convenience. Zemčík et al. [10] developed a linear 4-node element with the DSG method implemented to resolve shear locking effects and EAS to handle the membrane locking effects. Yang et al. [11] presented a linear quadrilateral piezoelectric shallow shell element with the ANS technique, while Nguyen et al. [12] proposed a linear triangular shell element based on the DSG approach.

Besides the equivalent single-layer theories, layer-wise theories were also addressed in modeling of smart laminated structures. A number of those approaches rely on the Carrera Unified Formulation (CUF) for multilayered plates and shells [13]. Cinefra et al. [14] proposed a 9-node plate element that implements mixed interpolation of tensorial components (MITC) approach and variable through-the-thickness layer-wise kinematics to perform linear static analyses. This development was extended to free-vibration analyses of piezoelectric plates [15]. Milazzo [16] used both equivalent single-layer and layer-wise approaches for piezoelectric laminated plates whereby the coupled-field problem was reduced to mechanical one.

The theoretical contributions of Tzou [17] and numerical developments by Rabinovitch [18], Kulkarni and Bajoria [19], Lentzen et al. [20], Klinkel and Wagner [21] addressed the geometrically nonlinear effects in the behavior of smart thin-walled smart structures. However, 
so far this aspect was much less in the focus of the researchers compared to the developments for linear analysis; thus, further contributions would be worthwhile.

In the present work a recently developed linear 3-node shell element [22] is applied to resolve a linear and geometrically nonlinear dynamic response of piezoelectric laminated shells. The basic features of the element are briefly described and several dynamic linear and nonlinear sensor and actuator cases are considered to verify the applicability of the developed element formulation by comparing the obtained results with the solutions from the available literature.

\section{FeAtures of THE LiNEAR PIEZOELECTRIC SHELl ELEMENT}

Only the most important features of the triangular piezoelectric shell element, which is used in this work, are presented here. A detailed element formulation can be found in [22].

The element uses five mechanical degrees of freedom, three translations and two rotations, per node and, in addition, as many electrical degrees of freedom as piezolayers. The electrical degrees of freedom are the differences of electric potentials between the electrodes of a piezolayer.

The mechanical field of the element is enhanced by the Cell Smoothed - Discrete Shear Gap (CS-DSG) formulation. The Mindlin-Reissner kinematical assumptions are implemented and, hence, the transverse shear effects are included. The discrete shear gap technique proposed by Bletzinger [6] is implemented to alleviate the transverse shear locking. The strain smoothing technique suggested by Nguyen et al. [12] is applied to improve the accuracy and stability of the element, and, furthermore, to render the element formulation independent of the node numbering sequence.

Two different coordinate systems presented in Fig. 1 are used within the formulation: global $(\mathrm{x}, \mathrm{y}, \mathrm{z})$ and local $\left(\mathrm{x}^{\prime}, \mathrm{y}^{\prime}, \mathrm{z}^{\prime}\right)$. The structural displacement field is given with respect to the global coordinate system that is fixed in space.

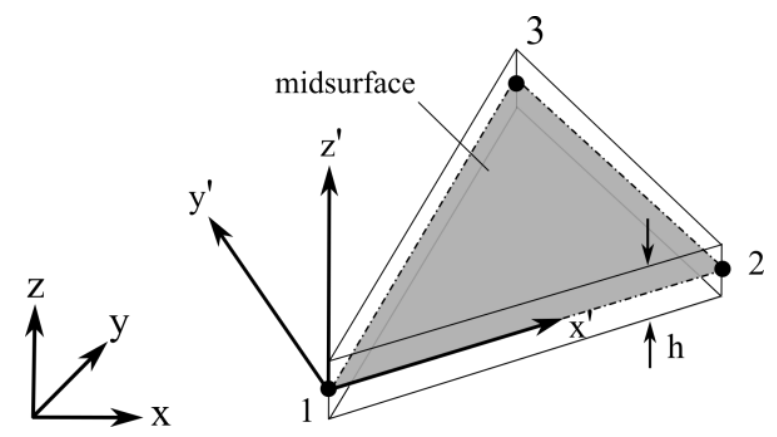

Fig. 1 Geometry and coordinate systems of the 3-node shell element

The local element coordinate system $\left(\mathrm{x}^{\prime}, \mathrm{y}^{\prime}, \mathrm{z}^{\prime}\right)$ is used to derive the mechanical strain and stress fields as well as the electro-mechanical coupling.

Regarding the piezoelectric layers, they are assumed to operate by using the piezoelectric $\mathrm{e}_{31}$-effect, which implies that the in-plane strain field is coupled to the electric field acting in the thickness direction. Electric field $E$ within the piezoelectric layers is assumed to be 
constant, which leads to a linear distribution of electric potential across thickness $\varphi$ so that the following relations hold:

$$
\mathrm{E}=-\frac{\partial \varphi}{\partial \mathrm{z}^{\prime}} \Rightarrow \mathrm{E}_{\mathrm{k}}=-\frac{\Phi_{\mathrm{k}}}{\mathrm{h}_{\mathrm{k}}}
$$

where $\Phi_{k}$ is the difference of electric potentials between the electrodes and $h_{k}$ is the layer thickness ( $\mathrm{k}$ in the subscript pertains to the layer number in the sequence of layers).

The element formulation is also extended to the geometrically nonlinear analysis. For this purpose the element-based co-rotational (CR) FE formulation [22, 23] is used, thus covering structural deformations characterized by the finite local rotations, whereby the strains remain small.

\section{FINITE ELEMENT EQUATIONS}

The coupled electro-mechanical dynamic FE equations may be derived using the Hamilton's principle for a piezoelectric continuum [24]. The FE system of equations for a geometrically nonlinear dynamic analysis by means of an implicit time integration scheme reads:

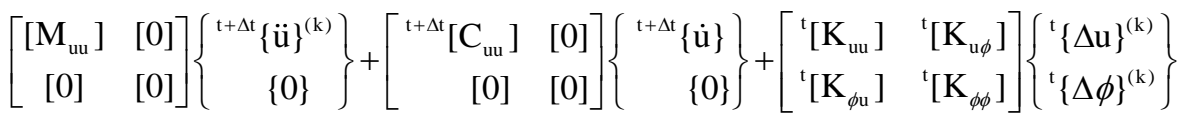

$$
\begin{aligned}
& =\left\{\begin{array}{c}
{ }^{\mathrm{t}+\Delta \mathrm{t}}\left\{\mathrm{F}_{\mathrm{ext}}\right\}-{ }^{\mathrm{t}+\Delta \mathrm{t}}\left\{\mathrm{F}_{\mathrm{in}}\right\}^{(\mathrm{k}-1)} \\
{ }^{\mathrm{t}+\Delta \mathrm{t}}\left\{\mathrm{Q}_{\mathrm{ext}}\right\}-{ }^{\mathrm{t}+\Delta \mathrm{t}}\left\{\mathrm{Q}_{\mathrm{in}}\right\}^{(\mathrm{k}-1)}
\end{array}\right\}
\end{aligned}
$$

where $\left[\mathrm{M}_{\mathrm{uu}}\right]$ is the mass matrix, $\left[\mathrm{C}_{\mathrm{uu}}\right]$ the damping matrix, $\left[\mathrm{K}_{\mathrm{uu}}\right],\left[\mathrm{K}_{\mathrm{u} \phi}\right],\left[\mathrm{K}_{\phi \mathrm{u}}\right]$ and $\left[\mathrm{K}_{\phi \phi}\right]$ are mechanical stiffness, piezoelectric direct and inverse coupling, and dielectric stiffness matrices, respectively, while vectors $\{\Delta \phi\},\{\Delta \mathrm{u}\},\{\dot{\mathrm{u}}\},\{\ddot{\mathrm{u}}\}$ comprise the incremental differences of electric potentials of the piezolayers, incremental displacements, nodal velocities and accelerations, respectively. Vectors $\left\{F_{\text {ext }}\right\},\left\{F_{i n}\right\},\left\{Q_{\text {ext }}\right\}$ and $\left\{Q_{\text {in }}\right\}$ on the right hand-side of the FE equations are external and internal mechanical forces and electric charges, respectively. Index $\mathrm{k}$ in the superscript denotes the iteration number.

Rayleigh damping is used to introduce the dissipative effects in the FE equations. It consists of stiffness and mass proportional terms:

$$
\left[\mathrm{C}_{\mathrm{uu}}\right]=\alpha\left[\mathrm{K}_{\mathrm{uu}}\right]+\beta\left[\mathrm{M}_{\mathrm{uu}}\right]
$$

where $\alpha$ and $\beta$ are the Rayleigh damping coefficients [25].

\section{NUMERICAL EXAMPLES}

In what follows, a set of examples is studied to demonstrate the applicability of the element for linear and geometrically nonlinear dynamic analysis of smart thin-walled structures. The considered structures are made of composite laminates with various combinations of fiber-reinforced, isotropic and piezoelectric layers. The properties of all 
the used materials are given in Table 1, where Y denotes the Young's modulus and $v$ the Poisson's ratio (with indices referring to the material orientation). The values in empty cells of Table 1 are considered to be equal to zero in the studied examples. The thickness and stacking sequence of layers vary in the examples and will be specified for each example separately.

Table 1 Layers material properties (given in principal material directions)

\begin{tabular}{lccccccc}
\hline & T300/976 & Aluminum & Steel & PTZ-4 & Gr/Ep & PIC 151 & PTZ \\
\hline $\mathrm{Y}_{11}[\mathrm{Gpa}]$ & 150.0 & 70.3 & 210 & 81.3 & 132.28 & 61.0 & 63.0 \\
$\mathrm{Y}_{22}[\mathrm{Gpa}]$ & 9.0 & 70.3 & 210 & 81.3 & 10.76 & 61.0 & 63.0 \\
$\mathrm{Y}_{33}[\mathrm{Gpa}]$ & 9.0 & 70.3 & 210 & 64.5 & 10.76 & 48.4 & 63.0 \\
$\mathrm{v}_{12}[-]$ & 0.3 & 0.345 & 0.3 & 0.33 & 0.24 & & 0.3 \\
$\mathrm{v}_{13}[-]$ & 0.3 & 0.345 & 0.3 & 0.43 & 0.24 & & 0.3 \\
$\mathrm{v}_{23}[-]$ & 0.3 & 0.345 & 0.3 & 0.43 & 0.49 & & 0.3 \\
\hline Density $\left[\mathrm{kg} / \mathrm{m}^{3}\right]$ & & & & & & & \\
& 1600 & 2690 & 7800 & 7600 & 1578 & 7760 & 7600 \\
\hline $\begin{array}{l}\text { Piezoelectric constants } \\
\mathrm{e}_{31}=\mathrm{e}_{32}\left[\mathrm{Cm}^{-2}\right] \\
\text { Dielectric constant }\left[\mathrm{F} \mathrm{m}^{-1}\right]\end{array}$ & & & & & & \\
$\quad \mathrm{d}_{31}\left(\times 10^{-8}\right)$ & & & -14.8 & & 9.6 & -22.87 \\
\hline
\end{tabular}

The examples include both actuator and sensor cases. In the actuator case the piezopatches are subjected to a predefined electric voltage, thus causing mechanical excitation due to the inverse piezoelectric effect. In the linear analysis the computation of induced mechanical loads ${ }^{t}\left\{F_{\phi, e}\right\}$ is performed on the element level as follows:

$$
{ }^{\mathrm{t}}\left\{\mathrm{F}_{\phi, \mathrm{e}}\right\}=\left[\mathrm{K}_{\mathrm{u} \phi, \mathrm{e}}\right]{ }^{\mathrm{t}}\left\{\phi_{\mathrm{a}, \mathrm{e}}\right\}
$$

where the matrices and vectors are defined on the element level. In the nonlinear analysis the system matrices, including the piezoelectric coupling terms, have to be updated first. In the framework of the CR-formulation, the element piezoelectric coupling matrix is updated using element rotation matrix ${ }^{\mathrm{t}}\left[\mathrm{R}_{\mathrm{e}}\right]$ :

$$
{ }^{\mathrm{t}}\left[\mathrm{K}_{\mathrm{u \phi}, \mathrm{e}}\right]={ }^{\mathrm{t}}\left[\mathrm{R}_{\mathrm{e}}\right]{ }^{0}\left[\mathrm{~K}_{\mathrm{u \phi}, \mathrm{e}}\right]
$$

In the sensor case, the direct piezoelectric effect is used to induce electric voltage ${ }^{\mathrm{t}}\left\{\phi_{\mathrm{s}, \mathrm{e}}\right\}$ (again, computed on the element level) due to the external mechanical loads, whereby the external electric charges are equal to zero:

$$
{ }^{\mathrm{t}}\left\{\phi_{\mathrm{s}, \mathrm{e}}\right\}=-\left[\mathrm{K}_{\phi \phi, \mathrm{e}}\right]^{-1}\left[\mathrm{~K}_{\phi \mathrm{u}, \mathrm{e}}\right]{ }^{\mathrm{t}}\left\{\mathrm{u}_{\mathrm{e}}\right\}
$$

where, again, all the vectors and matrices are defined on the element level (index 'e'). In the linear analysis the above equation is used directly, whereas in the geometrically nonlinear analysis the rotation-free (i.e. purely deformational) displacements are computed first. As a sensor patch/layer is discretized by a number of finite elements, a constraint is introduced that the induced electric voltages in the sensor layer are equal in all those elements. In this manner, the obtained sensor voltage reflects the average value of the in-plane strains caused in the sensor layer by the action of external mechanical loads. 


\subsection{Modal analysis of a simply supported piezoelectric plate}

In the first case a modal analysis of a composite piezoelectric plate is performed. In order to verify the CS-DSG3 formulation and to illustrate the influence of the electromechanical coupling on the dynamic properties two cases with different electric boundary conditions are investigated. In the first one, the electrodes of the piezolayers are shortcircuited (SC). Hence, the electric potential $\{\phi\}$ is equal to zero. This leads to the purely mechanical eigenvalue problem, i.e. the natural frequencies and modes are the same as if only purely mechanical field was considered.

In the second case, the electrodes are assumed to be open $(\mathrm{O})$ which implies zero electric charge as a boundary condition. From Eq. (2) follows:

$$
\left\{\phi_{\mathrm{s}}\right\}=-\left[\mathrm{K}_{\phi \phi}\right]^{-1}\left[\mathrm{~K}_{\phi \mathrm{u}}\right]\{\mathrm{u}\}
$$

Hence, an electric potential difference is generated in the sensor layer if the shell is deformed. Due to the open electrodes, the electric voltage induces mechanical stresses through an inverse piezoelectric effect. In the modal analysis these stresses are taken into account by a modified stiffness matrix obtained by substituting $\left\{\phi_{\mathrm{s}}\right\}$ into Eq. (2):

$$
\left[\mathrm{K}^{*}\right]=\left[\mathrm{K}_{\mathrm{uu}}\right]+\left[\mathrm{K}_{\mathrm{u} \phi}\right]\left[\mathrm{K}_{\phi \phi}\right]^{-1}\left[\mathrm{~K}_{\phi \mathrm{u}}\right]
$$

The electro-mechanical coupled eigenvalue problem reads then:

$$
\left[\left[\mathrm{K}^{*}\right]-\omega^{2}\left[\mathrm{M}_{\mathrm{uu}}\right]\right]\{\mathrm{u}\}=0
$$

It is obvious that the natural frequencies and mode shapes are in this case influenced by the properties of the piezoelectric material. The natural frequencies are increased in the open electrodes case compared to the short-circuited case because of the additional stiffness term.

Both the cases are studied on the same structure, at all edges simply supported square laminated piezoelectric plate (dimensions $\mathrm{a} \times \mathrm{a}=0.2 \times 0.2 \mathrm{~m}$, see Fig. 2 ).

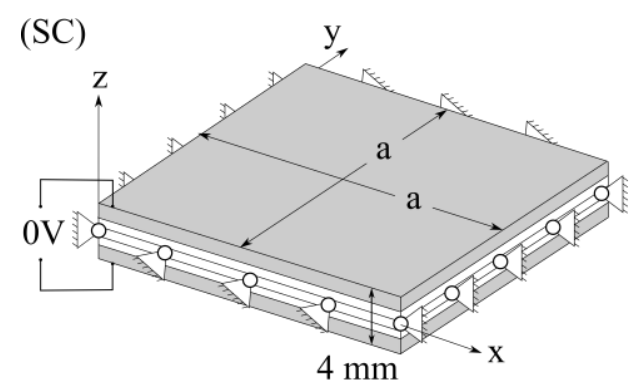

$4 \mathrm{~mm}$

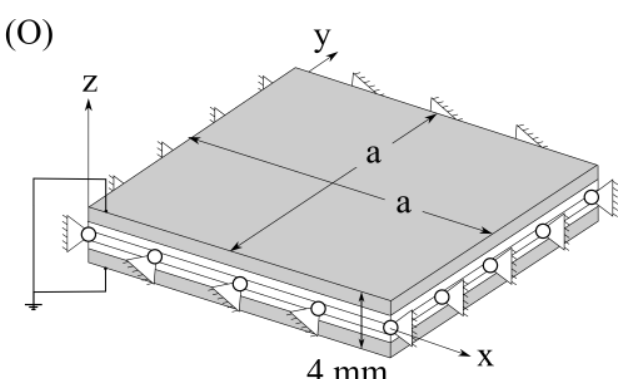

$4 \mathrm{~mm}$

Fig. 2 Geometry of the simply supported plate with different electric boundary conditions (SC) and (O)

The composite ply layup is $\left[\mathrm{p} / 0^{\circ} / 90^{\circ} 0^{\circ} / \mathrm{p}\right]$. The outer layers are made of piezoelectric PTZ-4 ceramics and the composite layers of Graphite Epoxy $(\mathrm{Gr} / \mathrm{Ep})$. The thickness of each piezoelectric layer is $0.0004 \mathrm{~m}$ and each composite layer is $0.001068 \mathrm{~m}$ thick. Saravanos [26] computed the first natural frequency for this structure using different 
meshes. These results are used for the comparison with the current formulation. In order to make the results comparable to [26] the value of density of all layers is set to one $\mathrm{kg} / \mathrm{m}^{3}$. Table 2 shows the result convergence for the first natural frequency determined by using three different meshes (32, 128 and 288 elements). For an easier comparison these results are normalized with respect to a reference solution. For the SC-case the reference solution is obtained with Abaqus using a $24 \times 24$ elements mesh and the biquadratic S8 element while the reference solution of case $(\mathrm{O})$ is analytical and presented in [26]. The difference to the reference solutions is in both cases less than $0.5 \%$ for the mesh with 288 elements.

Table 2 The normalized first eigenfrequency - convergence analysis

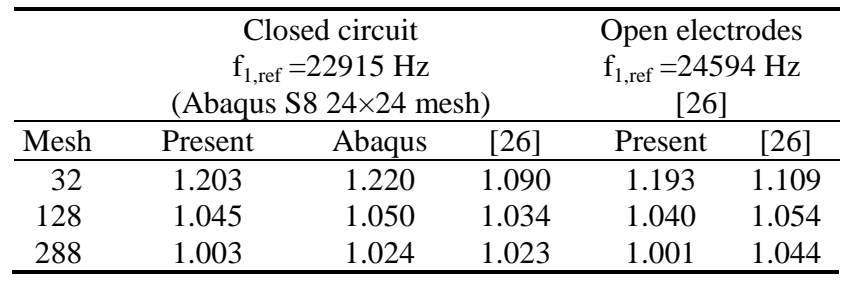

\subsection{Transient analysis of an active beam structure (linear dynamic actuator case)}

The undamped dynamic behavior of a clamped beam with two pairs of piezopatches bonded onto its outer surfaces is studied in this example. The beam geometry is depicted in Fig. 3. It is made of aluminum, while the piezopatches are made of PIC 151 (Table 1).

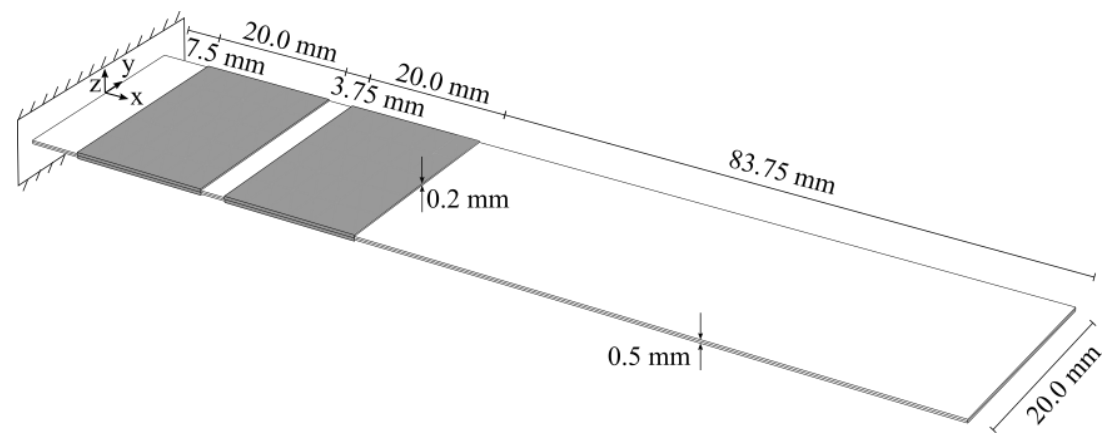

Fig. 3 Geometry of the active beam structure with two pairs of piezopatches

The oppositely polarized piezopatches are subjected to a time-varying voltage. The voltage is a sinusoidal function with amplitude of $100 \mathrm{~V}$ and frequency of $100 \mathrm{~Hz}$. This induces time-varying bending moments with respect to the structure's mid-surface, which are uniformly distributed over the patch edges. The resulting transverse beam tip deflection (w) is observed in a time interval of $0.1 \mathrm{~s}$ with constant time-step of $0.0001 \mathrm{~s}$ (1000 steps) using the Newmark time integration scheme [25]. The first three eigenfrequencies of the beam considered as a purely mechanical structure are $31.1 \mathrm{~Hz}, 131.8 \mathrm{~Hz}$ and $349.9 \mathrm{~Hz}$. Hence the answer of the structure subjected to an excitation with the frequency of $100 \mathrm{~Hz}$ is dominated by the first 
two natural mode shapes. The transient analysis is carried out using a FE mesh with 320 elements, which yielded a converged solution for the first three eigenfrequencies and mode shapes. For the purpose of verification, a transient analysis of the same structure was computed in Abaqus using the S3 shell element, the same mesh and time-step, whereby the equivalent mechanical nodal excitations were pre-computed and directly applied. The obtained time histories of the tip deflection are shown in Fig. 4. The results of the present formulation are in a very good agreement with those from Abaqus.

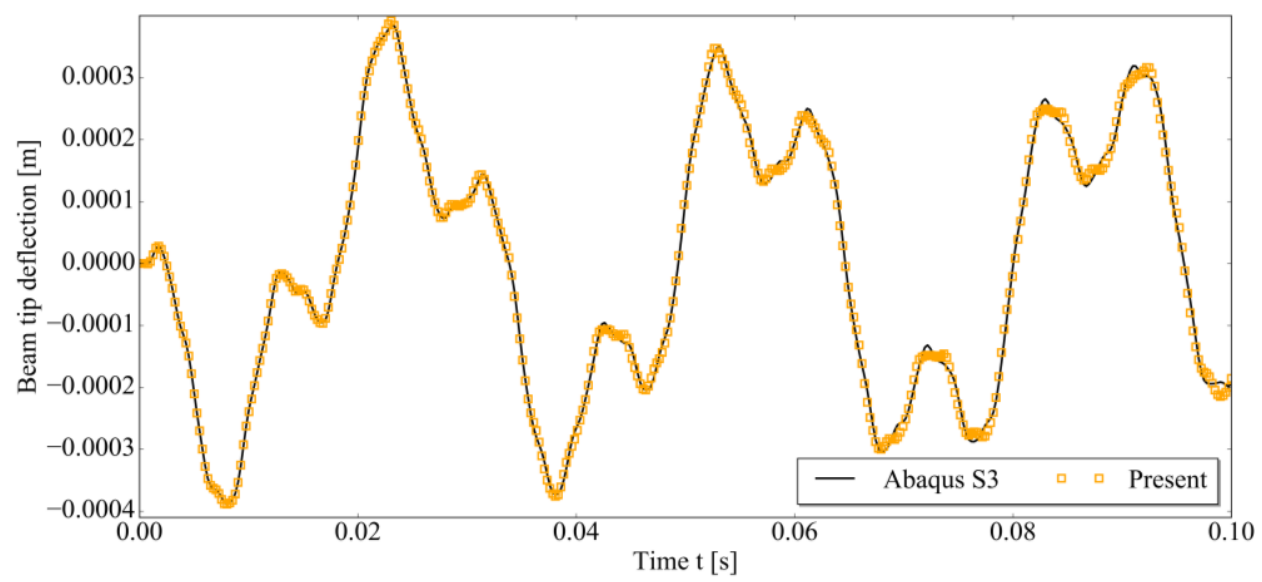

Fig. 3 Linear dynamic behavior of the active beam (1000 steps)

\subsection{Nonlinear dynamic analysis of a two-edge-simply-supported laminate}

The previous example was computed using the assumption of linearity. Hence, the structural stiffness and induced piezoelectric loads were calculated using the initial configuration as a reference configuration. This example will be calculated using both the assumption of linearity and a geometrically nonlinear approach. The geometry of the laminate composite plate simply supported over two shorter parallel edges is shown in Fig. 5. The laminate consists of three layers. The aluminum mid-layer is $0.5 \mathrm{~mm}$ thick and each outer PTZ-4 layer has a thickness of $0.25 \mathrm{~mm}$.

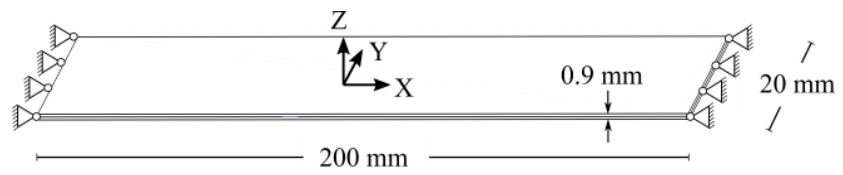

Fig. 5 Geometry of the two-edge-simply-supported structure

The same type of excitation from the previous case is used here as well. It is the timevariable electric voltage with amplitude of $100 \mathrm{~V}$ and frequency of $100 \mathrm{~Hz}$. The response of the structure is computed for a time interval of $0.1 \mathrm{~s}$ with a constant time-step of $0.0001 \mathrm{~s}$ using the Newmark scheme. The comparison between linear and nonlinear dynamic response is obvious in Fig. 6. It shows that, even in the range of relatively small deformations, the linear and the geometrically nonlinear response could differ significantly. Such a result emphasizes the 
necessity of taking into account nonlinear effects. The geometrically nonlinear computation is verified by means of Abaqus. As already mentioned in the previous example, the equivalent mechanical excitation is first pre-computed and then directly applied in Abaqus. It should be emphasized that the induced bending moments are of the follower type as their orientation depends on the current structural configuration, and this is how they are defined in Abaqus (the option 'follow nodal rotation' was used). Again, observing the structure's mid-point deflection, practically congruent geometrically nonlinear results obtained by means of the developed element and in Abaqus can be seen in Fig. 6.

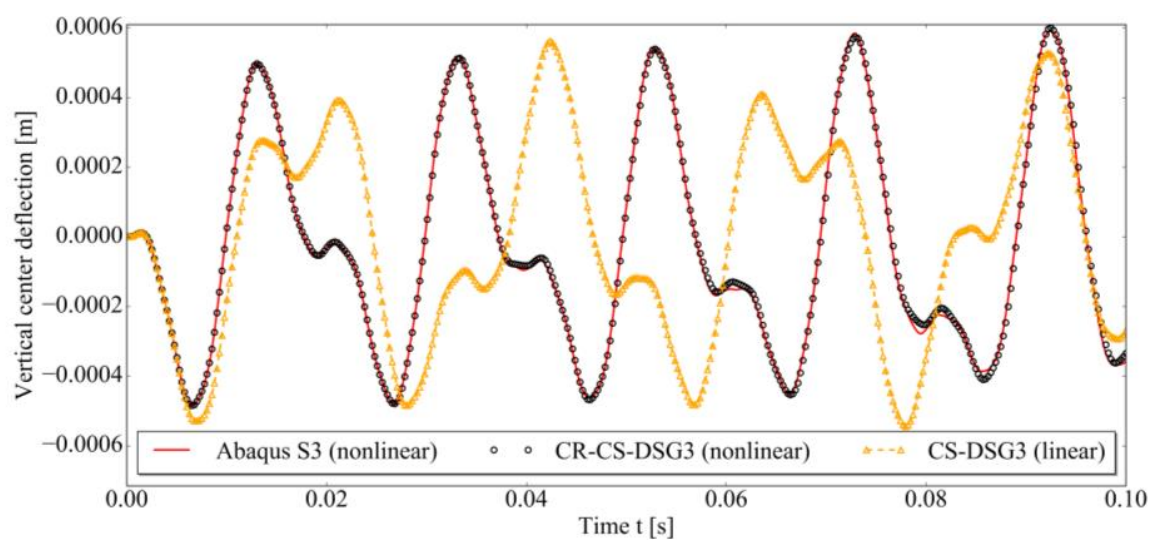

Fig. 6 Two-edge-simply supported structure under harmonic excitation

\subsection{Clamped piezoelectric plate (linear dynamic sensor case)}

A composite plate clamped over one edge is considered next. The plate geometry is shown in Fig 7. The composite consists of six layers. The outer two are oppositely polarized PTZ layers and the remaining four are T300/976 plies. Each T300/976 layer has a thickness of 0.25 $\mathrm{mm}$ and each PTZ layer is $0.1 \mathrm{~mm}$ thick. The antisymmetric composite stacking sequence is $\left[\mathrm{p} /-45^{\circ} / 45^{\circ} /-45^{\circ} / 45^{\circ} / \mathrm{p}\right]$ with respect to the global $\mathrm{x}$-axis. This structure has been already considered in the available literature $[27,28]$ as a static linear actuator case. For this reason, the exact same static case will be computed here first. After that, a dynamic sensor case will be addressed.

In the linear static case a uniform surface load $\mathrm{p}=100 \mathrm{~N} / \mathrm{m}^{2}$ acts upon the plate. Both PTZ layers are used as actuators subjected to three different voltages: $0 \mathrm{~V}, 30 \mathrm{~V}$ and $50 \mathrm{~V}$.

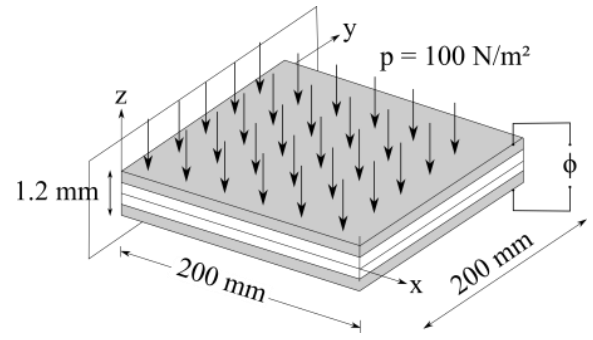

Fig. 7 Clamped piezoelectric plate subjected to uniform surface load 
The shape of the plate centerline is computed using a mesh of 200 elements. The comparison between the obtained results and the solutions of Lam et al. [27] and Zhang [28] shows a rather good agreement. For the sake of better readability, only the results of Lam et al. [27] and those obtained by the present formulation are presented in Fig. 8.

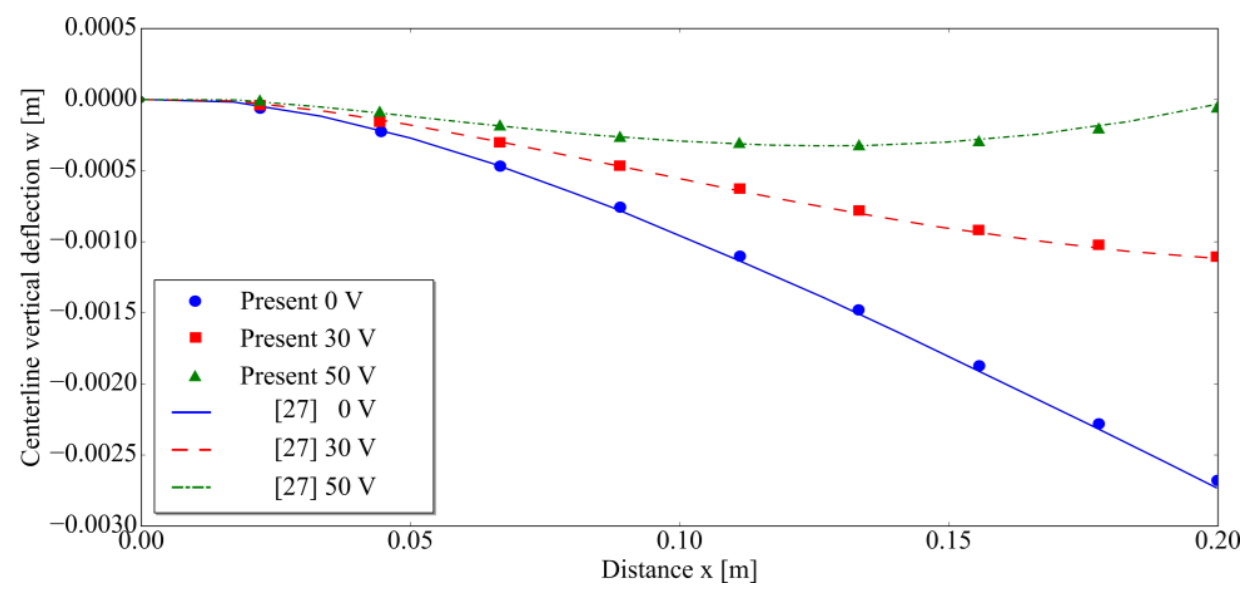

Fig. 8 Centerline deflection subjected to uniform load and different input voltages

In the linear dynamic analysis, the piezolayers are used as sensors and the composite plate is subjected to harmonic varying concentrated force. The force acts at point A (see Fig. 9) with an amplitude of $0.2 \mathrm{~N}$ and frequency of $1 \mathrm{~Hz}$. The induced sensor voltage of the lower layer is observed in a period of $4 \mathrm{~s}$ with a time-step of $0.005 \mathrm{~s}$ using the Newmark scheme and the same mesh as in the previous static analysis.

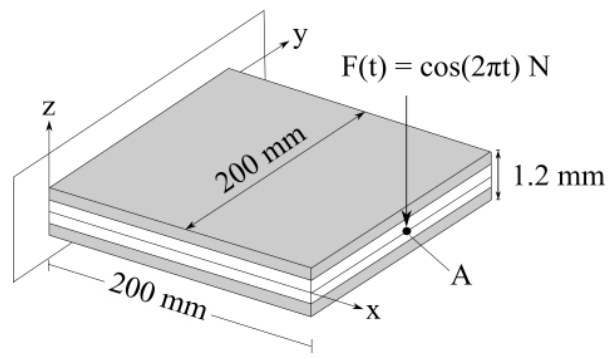

Fig. 9 Clamped piezoelectric plate subjected to harmonic varying concentrated force

Zhang et al. [29] studied this example using the SH851URI biquadratic shell element (uniformly reduced integration) along with the modal superposition method using the first 12 modes. Fig. 10 shows a good agreement in the amplitude and frequency of the sensor potential response between the current formulation and Zhang et al. [29]. The minor local differences are attributed to a different time-step (not specified in [29]) and a different damping definition. 


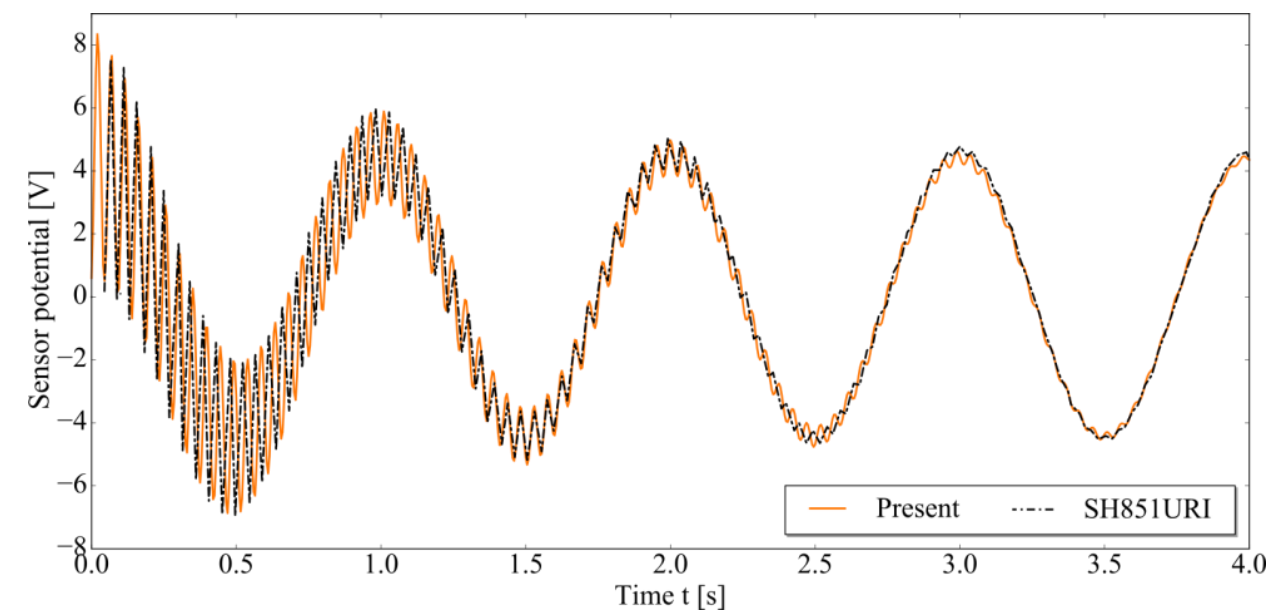

Fig. 10 Dynamic sensor response of piezoelectric plate under harmonic concentrate force

\subsection{Simply supported piezoelectric plate (nonlinear dynamic sensor case)}

The next example illustrates the influence of geometrically nonlinear effects on the dynamic sensor response when piezoelectric plate structures are considered. Fig. 11 shows the plate geometry together with the boundary conditions. The laminate consists of three layers. The outer two are oppositely polarized piezoelectric PTZ layers with a thickness of $0.1 \mathrm{~mm}$, while the mid-layer is $0.5 \mathrm{~mm}$ thick and made of steel. The plate is discretized so that the FE mesh consists of 512 elements. The plate is subjected to a concentrated force with periodic time dependent amplitude (see Fig. 11). The vertical displacement of point B (see Fig. 10) and sensor response of the upper (1) and lower (2) layers is observed in a time period of $0.2 \mathrm{~s}$ using a time-step of $0.005 \mathrm{~s}$.

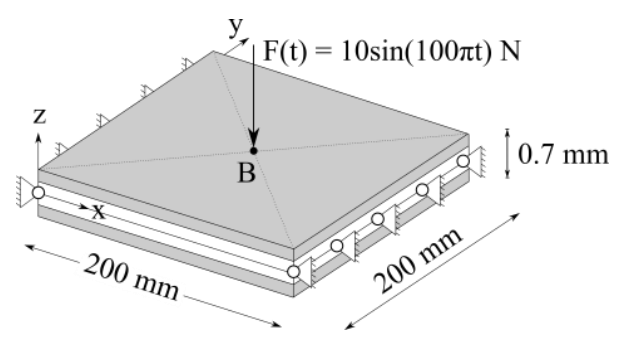

Fig. 11 Simply supported piezoelectric plate geometry

In the first step the dynamic response of the structure is determined with Abaqus using the same mesh and time-step. Fig. 12 shows the vertical deflection of point B computed as linear and geometrically nonlinear dynamic response. Again, a good agreement between the results from Abaqus and the present formulation can be noticed. 


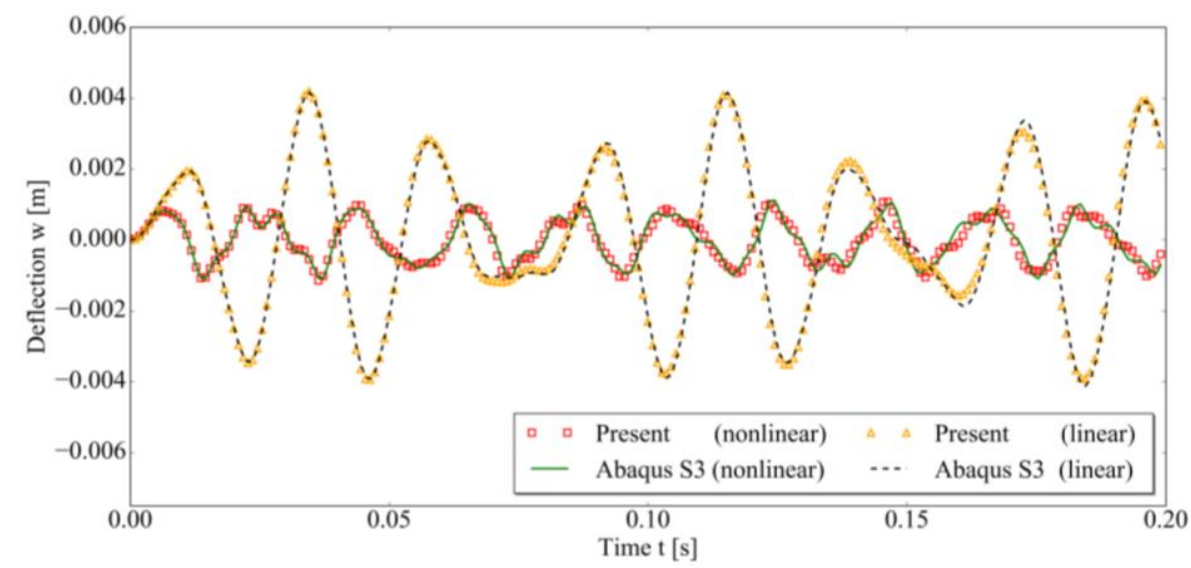

Fig. 12 Vertical deflection of a simply supported piezoelectric plate under harmonic excitation

In the second step, the linear and nonlinear sensor voltage response is computed for the same test case. The obtained results for the upper (1) and lower (2) piezoelectric layers are presented in Fig. 13. In the linear analysis, the stiffness matrix is determined for the initial configuration and the mechanical excitation leads to bending deformation. As a result of the opposite polarization of the PTZ layers, the computed sensor voltages of the layer (1) and (2) are equal (see Fig. 13).

In the nonlinear analysis the stiffness matrix changes continuously with the structural deformation. The deformation involves membrane and bending effects and, consequently, the sensor voltages of the upper and lower layer differ. The difference between the linear and the nonlinear results depends on the boundary and loading conditions. In this case, one can easily notice the differences in periods and amplitudes of the linear and nonlinear sensor response, demonstrating the importance to account for the geometrically nonlinear effects for adequate accuracy.

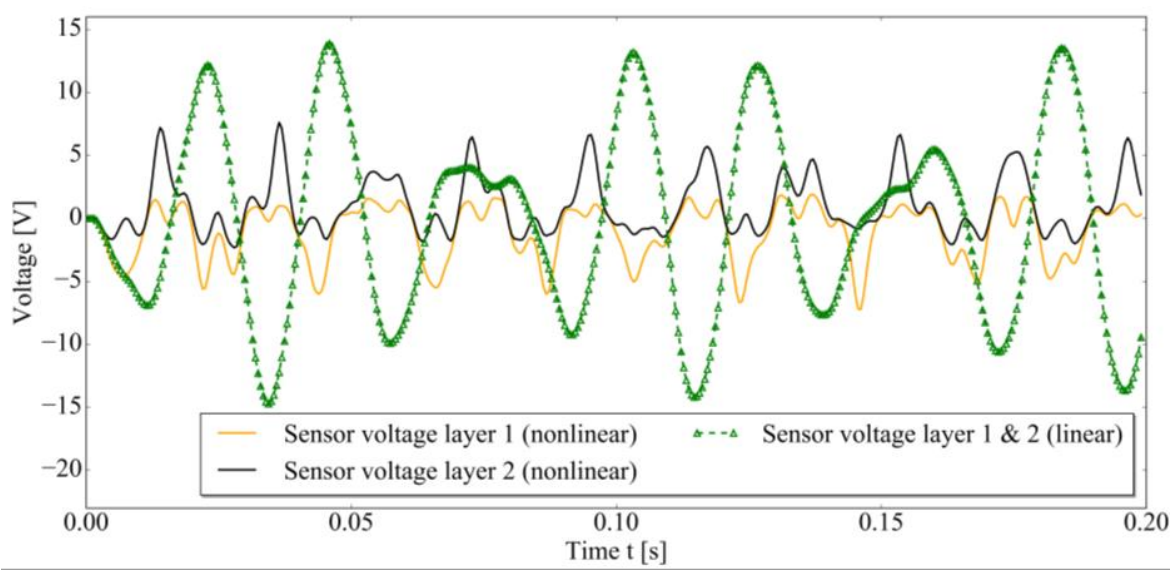

Fig. 13 Sensor voltage response of a simply supported piezoelectric plate under harmonic excitation 


\section{CONCLUSIONS}

The dynamics is of particular importance for smart structures as their advantages are quite often used to achieve active vibration suppression, radiated noise attenuation, etc. Simulation of the smart structures dynamic behavior is a significant prerequisite for their successful design, including control laws, i.e. algorithms that define the control strategy. The recently developed linear triangular shell type finite element [22] was used in this paper to perform dynamic analyses of thin-walled piezoelectric laminated structures. Both linear and geometrically nonlinear computations were performed. Actuator and sensor cases were considered. For the nonlinear computations, the co-rotational FE formulation was used. The verification of the results was done using either results available in the literature or the results from Abaqus by properly prepared equivalent mechanical models. A high agreement of the results validates the developed element. Furthermore, the geometrically nonlinear examples demonstrate that, depending on the boundary and loading conditions, the nonlinear effects can play a significant role even when relatively small deformations are caused. This is particularly valid for thin-walled structures.

\section{REFERENCES}

1. Benjeddou, A., 2000, Advances in piezoelectric finite element modeling of adaptive structural elements: a survey, Computers and Structures, 76, pp. 347-363.

2. Lee, S., Cho, B.C., Park, H.C., Goo, N.S., Yoon, K.J., 2004, Piezoelectric Actuator-Sensor Analysis using a Three-dimensional Assumed Strain Solid Element, Journal of Intelligent Material Systems and Structures, 15 , pp. 329-338.

3. Willberg, C., Gabbert, U., 2012, Development of a three-dimensional piezoelectric isogeometric finite element for smart structure applications, Acta Mechanica, 223, pp. 1837-1850.

4. Dvorkin, E.N., Bathe, K.J., 1984, A continuum mechanics based four-node shell element for general non-linear analysis, Engineering Computations, 1, pp. 77-88.

5. Huang, H.C., Hinton, E., 1986, A new nine node degenerated shell element with enhanced membrane and shear interpolation, International Journal for Numerical Methods in Engineering. 22(1), pp. 73-92.

6. Bletzinger, K.U., Bischoff, M., Ramm, E., 2000, A unified approach for shear-locking-free triangular and rectangular shell finite elements, Computers \& Structures, 75(3), pp. 321-334.

7. Marinkovic, D., Köppe, H., Gabbert, U., 2006, Numerically efficient finite element formulation for modeling active composite laminates, Mechanics of Advanced Materials and Structures, 13, pp. 379-392.

8. Marinković, D., Marinković, Z., 2012, On FEM modeling of piezoelectric actuators and sensors for thin-walled structures, Smart Structures and Systems, 9(5), pp. 411-426.

9. Nestorovic, T., Shabadi, S., Marinković, D., Trajkov, M., 2014, User defined finite element for modeling and analysis of active piezoelectric shell structures, Meccanica, 49(8), pp. 1763-1774.

10. Zemčík, B., Rolfes, R., Rose, M., Teßmer, J., 2007, High-performance four-node shell element with piezoelectric coupling for the analysis of smart laminated structures, International Journal for Numerical Methods in Engineering, 70(8), pp. 934-961.

11. Yang, Lammering, R., Mesecke-Rischmann, S., 2004, Advanced Shell Element Formulations for Coupled Electromechanical Systems Fan, Proc. Appl. Math. Mech., 4, pp. 386-387.

12. Nguyen-Thoi, T, Phung-Van, P., Thai-Hoang, C., Nguyen-Xuan, H., 2013, A Cell-based Smoothed Discrete Shear Gap method (CS-DSG3) using triangular elements for static and free vibration analyses of shell structures, International Journal of Mechanical Sciences, 74, pp. 32-45.

13. Carrera, E., 2003, Theories and finite elements for multilayered plates and shells: A unified compact formulation with numerical assessment and benchmarking, Arch. Comput. Meth. Engng, 10, pp. 215-296.

14. Cinefra, M., Carrera, E., Valvano, S., 2015, Variable Kinematic Shell Elements for the Analysis of Electro-Mechanical Problems, Mechanics of Advanced Materials and Structures, 22(1-2), pp. 77-106.

15. Cinefra, M., Valvano, S., Carrera, E., 2015, A layer-wise MITC9 finite element for the free-vibration analysis of plates with piezo-patches, International Journal of Smart and Nano Materials, 6(2), pp. 84-104. 
16. Milazzo, A., 2016, Unified formulation for a family of advanced finite elements for smart multilayered plates, Mechanics of Advanced Materials and Structures, 23(9), pp. 971-980.

17. Tzou, H.S., Bao, Y., 1997, Nonlinear piezothermoelasticity and multi-field actuations, part 1: Nonlinear anisotropic piezothermoelastic shell elements, Journal of Vibration and Acoustics, 119, pp. 374-381.

18. Rabinovitch, O., 2005, Geometrically nonlinear behavior of piezoelectric laminated plates, Smart Materials and Structures, 14(4), pp. 785-798

19. Kulkarni, S.A., Bajoria, K.M., 2007, Large deformation analysis of piezolaminated smart structures using higher-order shear deformation theory, Smart Materials and Structures, 16, pp. 1506-1516.

20. Lentzen, S., Klosowski, P., Schmidt, R., 2007, Geometrically nonlinear finite element simulation of smart piezolaminated plates and shells, Smart Materials and Structures, 16, pp. 2265-2274.

21. Klinkel, S., Wagner, W., 2008, A piezoelectric solid shell element based on a mixed variational formulation for geometrically linear and nonlinear applications, Computers and Structures, 86(1-2), pp. 38-46.

22. Rama, G., Marinković, D., Zehn, M., 2017, Efficient 3-node finite shell element for linear and geometrically nonlinear analysis of piezoelectric laminated structures, Journal of Intelligent Material Systems and Structures, accepted for publishing.

23. Marinković, D., Zehn, M., Marinković, Z., 2012, Finite element formulations for effective computations of geometrically nonlinear deformations, Advances in Engineering Software, 50, pp. 3-11.

24. Tiersten, H.F., 1969, Linear Piezoelectric Plate Vibrations, Springer, Plenum, New York.

25. Bathe, K.J., 1996, Finite element procedures in engineering analysis, Prentice Hall, Inc., Englewood Cliffs, New Jersey.

26. Saravanos, D.A., Heyliger, P.R., Hopkins D.A., 1996, Layerwise mechanics and finite element for the dynamic analysis of piezoelectric composite plates, Int. J. Solids Struct., 4, pp 359-78.

27. Lam, K.Y., Peng X.Q., Liu G.R., Reddy J.N., 1997, A finite-element model for piezoelectric composite laminates, Smart Mater. Struct., 6, pp. 583-591.

28. Zhang, S., 2014, Nonlinear FE Simulation and Active Vibration Control of Piezoelectric Laminated Thin-Walled Smart Structures, PhD thesis, Institute of General Mechanics RWTH Aachen University.

29. Zhang, S., Schmidt, R., Qin, X., 2015, Active vibration control of piezoelectric bonded smart structures using PID algorithm, Chinese Journal of Aeronautics, 28(1), pp. 305-313. 